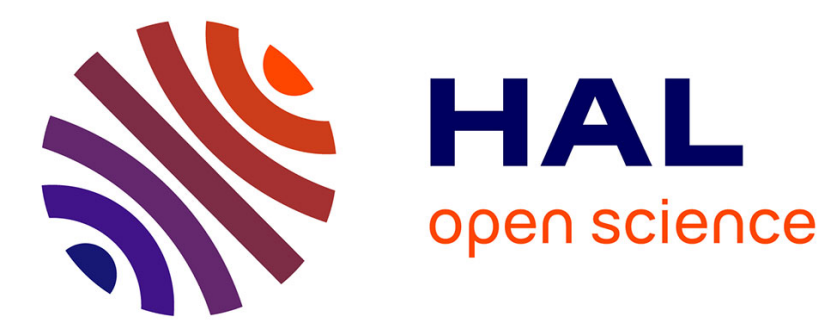

\title{
Quantum assignment for QoS-aware AFDX network with Deficit Round Robin
}

Aakash Soni, Jean-Luc Scharbarg, Jérôme Ermont

\section{To cite this version:}

Aakash Soni, Jean-Luc Scharbarg, Jérôme Ermont. Quantum assignment for QoS-aware AFDX network with Deficit Round Robin. 27th International Conference on Real Time Networks and Systems - RTNS 2019, Nov 2019, Toulouse, France. pp.70-79, 10.1145/3356401.3356421 . hal-02965546

\section{HAL Id: hal-02965546 \\ https://hal.science/hal-02965546}

Submitted on 13 Oct 2020

HAL is a multi-disciplinary open access archive for the deposit and dissemination of scientific research documents, whether they are published or not. The documents may come from teaching and research institutions in France or abroad, or from public or private research centers.
L'archive ouverte pluridisciplinaire HAL, est destinée au dépôt et à la diffusion de documents scientifiques de niveau recherche, publiés ou non, émanant des établissements d'enseignement et de recherche français ou étrangers, des laboratoires publics ou privés. 


\title{
Quantum assignment for QoS-aware AFDX network with Deficit Round Robin
}

\author{
Aakash Soni, Jean-Luc Scharbarg, Jérôme Ermont \\ University of Toulouse - IRIT - INPT/ENSEEIHT \\ Toulouse, France \\ firstname.lastname@enseeiht.fr
}

\begin{abstract}
Avionics Full Duplex switched Ethernet (AFDX) is the de facto standard for the transmission of critical avionics flows. It is a specific switched Ethernet solution based on First-in First-out (FIFO) scheduling. Timing constraints have to be guaranteed for such critical flows. The worst-case traversal time analysis introduces some pessimism, leading to a very lightly loaded network: typically less than $10 \%$ of the bandwidth is used. One solution to improve the utilisation of the network is to introduce Quality of Service (QoS) mechanisms. First, it can decrease worst-case delays for the most constrained avionics flows. Second less/non critical additional flows can be transmitted on the network with bounded impact on avionics ones. Deficit Round Robin (DRR) is such a QoS mechanism and it is envisioned for future avionics networks. An optimised WCTT analysis has been proposed for DRR on AFDX, based on network calculus. With DRR, the flow set is divided into classes and each class is allocated a quantum. In each round, transmissions are managed, based on these quanta. Thus delays are significantly impacted by quanta. The contribution of this paper is to propose an efficient quantum assignment for a set of critical avionics flow classes and at most one additional class with less/non critical flows.
\end{abstract}

\section{CCS CONCEPTS}

- Networks $\rightarrow$ Network performance modeling; Network performance analysis;

\section{KEYWORDS}

Quantum Allocation, Deficit Round Robin, WCTT analysis, AFDX, Switched Ethernet, Quality of Service.

\section{ACM Reference format:}

Aakash Soni, Jean-Luc Scharbarg, Jérôme Ermont. 2019. Quantum assignment for QoS-aware AFDX network with Deficit Round Robin. In Proceedings of 27th International Conference on Real-Time Networks and Systems, Toulouse, France, November 6-8, 2019 (RTNS 2019), 10 pages.

https://doi.org/10.1145/3356401.3356421

Permission to make digital or hard copies of all or part of this work for personal or classroom use is granted without fee provided that copies are not made or distributed for profit or commercial advantage and that copies bear this notice and the full citation on the first page. Copyrights for components of this work owned by others than ACM must be honored. Abstracting with credit is permitted. To copy otherwise, or republish, to post on servers or to redistribute to lists, requires prior specific permission and/or a fee. Request permissions from permissions@acm.org.

RTNS 2019, November 6-8, 2019, Toulouse, France

(C) 2019 Association for Computing Machinery.

ACM ISBN 978-1-4503-7223-7/19/11...\$15.00

https://doi.org/10.1145/3356401.3356421

\section{INTRODUCTION}

Up to now, Quality of Service (QoS) mechanisms are not used in practice in the context of avionics. The de facto standard is the AFDX network, which mainly implement a FIFO service discipline in switch output ports. Actually, two priority levels are available, but they are rarely used. Different approaches have been proposed for Worst-case traversal time analysis in the context of avionics, e.g. Network Calculus (NC) [2,3] with successful implementation to certify A380 and A350 AFDX backbone[1]. The pessimism of WCTT analysis as well as the fact that worst-case scenarios have a very low probability to occur lead to a very lightly loaded network. Typically, less than $10 \%$ of the available bandwidth is used for the transmission of avionics flows on an AFDX network embedded in an aircraft. One solution to improve the utilisation of the network is to introduce Quality of Service (QoS) mechanisms. Deficit Round Robin (DRR) is such a mechanism and it is envisioned for future avionics networks. The goals are, first to better use the bandwidth for avionics flows by assigning flows with very different timing constraints to different classes, second to allow the transmission of less/non critical additional flows.

Deficit Round Robin (DRR) was proposed in [10] to achieve fair sharing of the capacity of a server among several flow classes. It is based on quanta which are allocated to classes per round. The main interest of DRR is its simplicity of implementation. As long as specific allocation constraints are met, it can exhibit $O(1)$ complexity. A comparison of DRR scheduler with First-In-First-Out (FIFO) and Static Priority (SP) scheduler used in AFDX network is shown in [6]. The end-to-end delay (ETE) bounds are computed and the paper shows the comparatively better performance of DRR scheduler over FIFO and SP scheduler, given an optimised network configuration. Another DRR implementation is proposed in [4], which combines the DRR with SP scheduling, to improve schedulability and make more efficient use of hardware resources. A detailed analysis and improvement of DRR latency bound for homogeneous flows is given in [7]. Some mathematical errors of [7] are pointed out and corrected in [8]. Analysis of a server with DRR scheduler using NC method is first discussed in [5] which also proposes improvement in DRR latency. [5] generalises the analysis to network with heterogeneous flows. An optimised WCTT analysis for DRR on AFDX is proposed in [11].

The assignment of flows to classes and the quantum allocated to each class has a major impact on the end-to-end latencies of flows. In this paper, we assume that flows have been assigned to classes and we focus on credit allocation. This problem has been addressed in the context of non critical flows with Weighted Round Robin (WRR) [9]. To the best of our knowledge, no paper considered the situation of a network shared by critical and non critical flows. 
In this paper, we propose an efficient quantum allocation for an AFDX network implementing DRR and shared by a set of critical flow classes and at most one less/non critical flow class.

The rest of the paper is organised as follows. Section 2 summarises the context of the paper, i.e. the network model, DRR main features and worst-case analysis as well as the problem statement. Section 3 presents the proposed quantum assignment algorithm. Sections 4 and 5 illustrate the algorithm on a small example and evaluate it on a realistic case study. Section 6 concludes the paper and gives some direction for future works.

\section{CONTEXT}

In this section, we present the network model, which considers DRR scheduling, and we state the problem.

\subsection{Network Model}

In this paper, we consider an avionics switched Ethernet network (AFDX). It is composed of a set of end systems, interconnected by switches via full-duplex links.

End-systems are the source and destination of a set of flows. These flows are statically defined as virtual links (VL). They are forwarded by switches, based on a statically defined forwarding table. The forwarding process introduces a switching latency $(s l)$.

Each flow is shaped at the end system. A minimum interval $B A G_{i}$ is guaranteed between any two consecutive frames of flow $v_{i}$. Each flow $v_{i}$ is also constrained by a maximum frame length $\left(l_{i}^{\max }\right)$ and a minimum frame length $\left(l_{i}^{\text {min }}\right)$. The path $\mathcal{P}_{i}$ followed by a flow $v_{i}$ in the network is statically defined.

Figure 1 shows an example of an AFDX network. Four switches $\left(S_{1}\right.$ to $\left.S_{4}\right)$ interconnect nine end systems $\left(e_{1}\right.$ to $\left.e_{9}\right)$. Twenty flows $\left(v_{1}\right.$ to $\left.v_{20}\right)$ are transmitted on this network. Each switch output port has a set of buffers controlled by a Deficit Round Robin (DRR) scheduler. The maximum link bandwidth is $R=100 \mathrm{Mbits} / \mathrm{s}$. Flow features are given in Table 1.

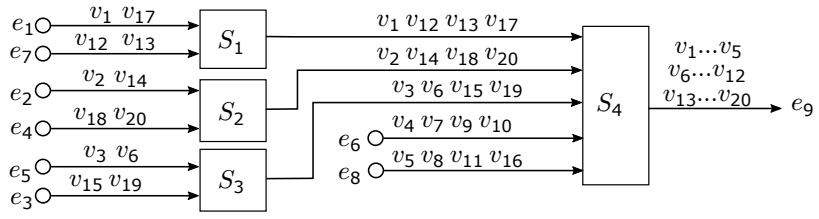

Figure 1: AFDX Network Example

In DRR scheduling, a set of queues is associated to each output port. Each queue belongs to a traffic class $C_{i}$. The flow frames are stored in queues corresponding to their traffic class. The DRR scheduler serves the queues in rounds. In a given queue, frames are served following a First Come First Served policy. The flows in Figure 1 are divided into three traffic classes $C_{1}, C_{2}$ and $C_{3}$. The classes $C_{1}$ and $C_{2}$ belongs to critical flows with an upper limit on delay constrains 3100 and $3200 \mu \mathrm{sec}$. The class $C_{3}$ belongs to non-critical flows with no delay constraints.
Table 1: VL parameters in network example (Figure 1)

\begin{tabular}{|c|c|c|c|c|c|}
\hline Flow & $\begin{array}{l}B A G_{i} \\
(\text { msec })\end{array}$ & $\begin{array}{c}l_{i}^{\max } \\
\text { (bytes) }\end{array}$ & $\begin{array}{c}l_{i}^{\min } \\
\text { (bytes) }\end{array}$ & $\begin{array}{c}\text { Delay } \\
\text { constraint } \\
(\mu \text { sec })\end{array}$ & Class \\
\hline$v_{1}, v_{2}, v_{3}$ & 8 & 1500 & 800 & \multirow{2}{*}{3100} & \multirow{2}{*}{$\begin{array}{c}\text { Critical } \\
\left(C_{1}\right)\end{array}$} \\
\hline$v_{4}, v_{5}$ & 8 & 990 & 800 & & \\
\hline$v_{12}, v_{13}, v_{18}$ & 16 & 1000 & 800 & \multirow{2}{*}{3200} & \multirow{2}{*}{$\begin{array}{c}\text { Critical } \\
\left(C_{2}\right)\end{array}$} \\
\hline$v_{20}, v_{15}, v_{19}$ & 16 & 990 & 800 & & \\
\hline$v_{17}, v_{14}, v_{6}, v_{7}$ & 32 & 1000 & 800 & \multirow{2}{*}{ - } & \multirow{2}{*}{$\begin{array}{l}\text { Non } \\
\text { critical } \\
\left(C_{3}\right)\end{array}$} \\
\hline $\begin{array}{l}v_{8}, v_{9}, v_{10} \\
v_{11}, v_{16}\end{array}$ & 32 & 990 & 800 & & \\
\hline
\end{tabular}

\subsection{Overview of DRR scheduler}

DRR scheduler was designed in [10] to achieve a better quality of service by fair sharing of available network bandwidth among the flows. Basically DRR is a variation of Weighted Round Robin (WRR) which allows fair sharing of bandwidth among variable length flow packets. DRR service is divided into rounds. In each scheduling round, active classes are served sequentially. A class is said to be active when it has some pending frames in its queue. Algorithm 1 shows DRR implementation at a switch output port $h$ serving $n$ traffic classes.

DRR scheduler assigns quantum $Q_{i}$ to each active class $C_{i}$. $Q_{i}$ represents the number of bytes allocated to $C_{i}$ in each round. The unused fraction of quantum in a given round is deficit $\Delta_{i}$ for the next round. Thus, the total amount of credit that $C_{i}$ can get in any round is $Q_{i}+\Delta_{i}$. Initially each class deficit is set to 0 (lines 1-3). Each active class queue is served in round robin order (lines 4-16). Empty queues are ignored in each round (line 6). The non-empty queues are credited by their quantum $Q_{i}$ added to the previous deficit $\Delta_{i}$ (line 7). Selected class can send frames as long as its queue is not empty and the deficit is larger than the head-of-line packet (line 8-12). On each transmission of frame, the credit is reduced by the frame size. If the queue becomes empty, the deficit is reset to 0 (lines 13-14). Then the next class is selected to be served.

Figure 2 illustrates DRR scheduling on the configuration in Figure 1. Each class is assigned a quantum of 1500 bytes. One frame of VLs $v_{1}, v_{3}, v_{5}, v_{6}, v_{7}, v_{12}, v_{13}, v_{14}, v_{17}, v_{18}, v_{20}$ with maximum frame size arrive at the output port of $S_{4}$ simultaneously and they are arbitrarily buffered in this order. We arbitrarily assume that class $C_{2}$ is selected first. Since its initial deficit is 0 , it gets a credit of 1500 (its quantum). Frame from $v_{12}$ is first in the queue and its size (1000 bytes) is less than the credit. Thus it is transmitted. Remaining credit is 500, which is not enough for the transmission of next $C_{2}$ pending frame (from $v_{13}$ ). Therefore, scheduler moves to class $C_{3}$ with a credit of 1500 . It allows the transmission of $v_{6}$ frame, but not $v_{7}$ one and the remaining credit is also 500. Then $C_{1}$ frame from $v_{5}$ is transmitted with a remaining credit of 510 . Then $C_{2}$ is selected again. It gets a credit of 2000 (500 of deficit plus 1500 of quantum). It allows the transmission of $v_{13}$ and $v_{18}$ frames, leading to a remaining credit of 0 , and so on. 


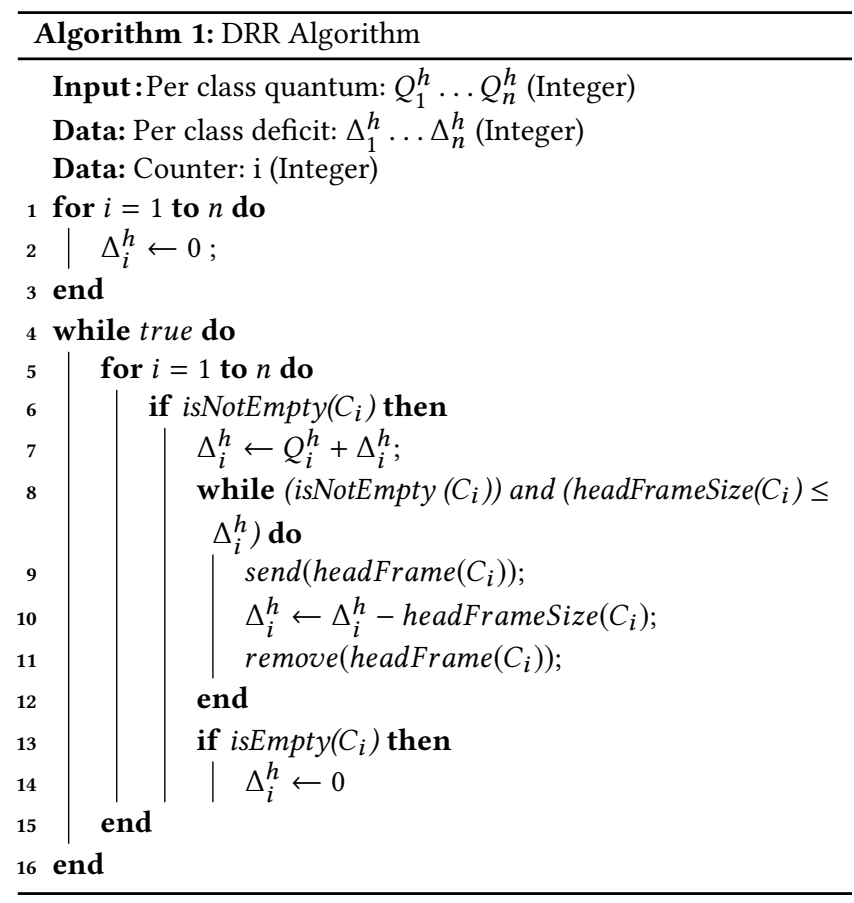

\subsection{DRR WCTT analysis}

A WCTT analysis is mandatory in order to guarantee that critical flows never exceed their deadline. Such a WCTT analysis has been proposed for DRR in [5] and optimised for AFDX in [11]. Both approaches are based on network calculus (NC). In this section, we give a very general overview of these approaches (only the details which are needed for the rest of the paper).

The NC theory is based on the (min, +) algebra [3]. It models the traffic and network elements by piecewise linear curves called arrival curves and service curves respectively.

The arrival curve of a flow represents an over-estimation of the traffic of this flow at any instant $t$. For a VL $v_{i}$ at its source end system $e s_{x}$, it is:

$$
\alpha_{i}^{e s_{x}}=\frac{l_{i}^{\max }}{B A G_{i}} \times t+l_{i}^{\max } \text { for } t>0 \text { and } 0 \text { otherwise }
$$

$l_{i}^{\text {max }}$ is the burst (one frame) while $\frac{l_{i}^{\max }}{B A G_{i}}$ is the long-term rate.

A frame of $v_{i}$ can experience jitter since it can be delayed by other frames before it arrives at a switch output port $h$. Thus the arrival curve of $v_{i}$ in $h$ is obtained by shifting the arrival curve of $v_{i}$ in $e s_{x}$ to the left by this jitter [2].

A switch output port $h$ with maximum service rate $R$ and switching latency $s l$ is modelled by a service curve:

$$
\beta^{h}(t)=R \times[t-s l]^{+}
$$

where $[a]^{+}$means $\max (a, 0)$.

In a DRR scheduler, the bandwidth is shared by all the classes at each output port $h$. Therefore each class $C_{x}$ receives a fraction $\rho_{x}^{h}$ of $R$, based on its assigned quantum $Q_{x}^{h}$ :

$$
\rho_{x}^{h}=\frac{Q_{x}^{h}}{\sum_{1 \leq j \leq n} Q_{j}^{h}} \times R
$$

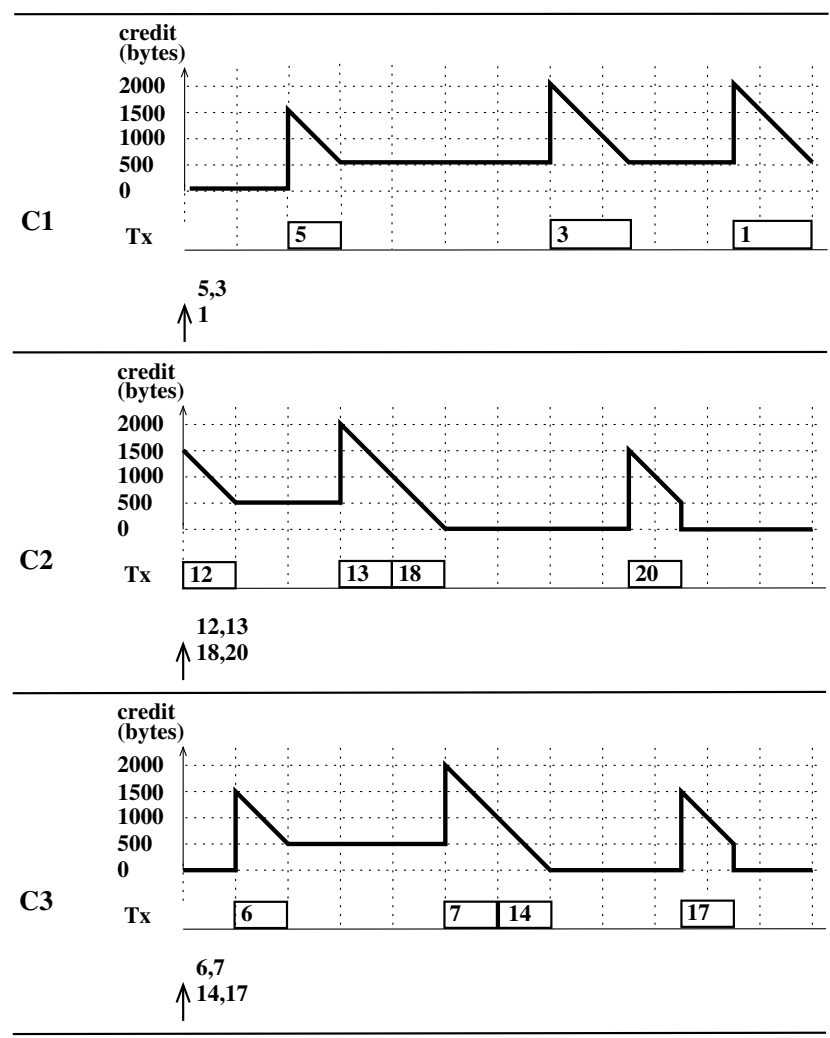

Figure 2: DRR rounds at $S_{4}$ output port

Moreover, class $C_{x}$ experiences the DRR scheduler latency $\Theta_{x}^{h}$ : it might have to wait before being served for the first time (while the other classes are served at their maximum capacity which is $\left.\sum_{j \neq x}\left(Q_{j}^{h}+\Delta_{j}^{\max , h}\right)\right)$ and, when it is served for the first time, it might get less than its allocated fraction $\rho_{x}^{h}$. This latency $\Theta_{x}^{h}$ can be illustrated with the example in Figure 2. Let us consider class $C_{1}$. Its first frame (from $v_{5}$ ) is served after one frame from $C_{2}$ and one frame from $C_{3}$, i.e. $160 \mu$ s. In the first round, frames from $v_{5}, v_{13}, v_{18}, v_{7}, v_{14}$ are served, leading to 5500 transmitted bytes. Thus $C_{1}$ gets $\frac{1500}{5500} \times 100=27.27 \mathrm{Mb} / \mathrm{s}$. Based on $\rho_{x}^{h}$, it should have received $33.33 \mathrm{Mb} / \mathrm{s}$.

Therefore the residual service curve for class $C_{x}$ at port $h$ is given by

$$
\beta_{x}^{h}(t)=\rho_{x}^{h} \times\left[t-\Theta_{x}^{h}-s l\right]^{+}
$$

The delay computation for class $C_{x}$ in each output port $h$ is bounded by the maximum horizontal difference between the overall arrival curve of $C_{x}$ flows in $h$ (sum of individual arrival curves) and the service curve for class $C_{x}$. These curves are illustrated in Figure 3. The service curve is an under-approximation of the actual staircase service curve of $C_{x}$. Indeed $C_{x}$ alternates periods when it get no service and periods when it gets full service at rate $R$. Such a staircase curve does not fit within NC, since it is not convex. The considered under-approximation is convex. 


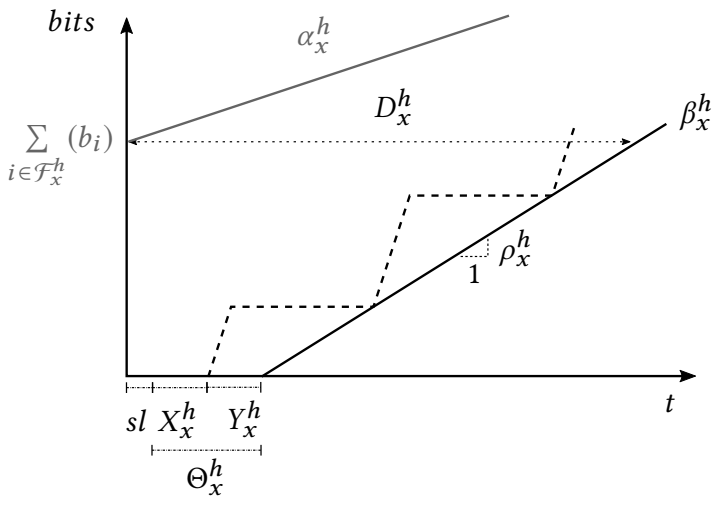

Figure 3: Arrival and Service Curves

The basic approach in [5] implement this delay computation. It does not make any assumption on the volume of competing traffic from other classes. It comes to consider that competing classes are always active. It might not be the case. Thus the approach in [11] optimises the one in [5] by quantifying the maximum amount of traffic of each competing class within the delay bound computed by [5].

\subsection{Problem statement}

Our goal is to allocate the quanta on an AFDX network implementing DRR scheduling so that:

- every critical frame respects its deadline,

- the delay of non critical frames is minimised.

Therefore the goal is to assign as few bandwidth as possible to critical classes in order to maximise the bandwidth assigned to the non critical class. As previously mentioned, we assume a set of $n-1$ critical classes $C_{1} \ldots C_{n-1}$ and one single non critical one $C_{n}$. We also assume that a given class is assigned the same quantum in all switch output ports. The quantum $Q_{x}$ assigned to a class $C_{x}$ has to allow the transmission of any frame of class $C_{x}$. Therefore, we must have

$$
Q_{x} \geq \operatorname{Max}_{x} \text { for } 1 \leq x \leq n
$$

where $\operatorname{Max}_{x}$ is the largest frame size among class $C_{x}$ frames.

If $\mathcal{V}_{x}$ is the set of class $C_{x}$ VLs, we have

$$
\operatorname{Max}_{x}=\max _{v_{j} \in \mathcal{V}_{x}} l_{j}^{\text {max }}
$$

In the next section we propose an algorithm that solves this quantum allocation problem. This algorithm assumes the basic WCTT computation in [5].

\section{QUANTUM ASSIGNMENT}

Since the quantum of a given class is the same in all switch ports, we omit node $h$ in notations in the rest of the paper.

Let us define $Q$ as the sum of quanta allocated to classes, i.e.

$$
Q=\sum_{1 \leq i \leq n} Q_{i}
$$

If we focus on one class $C_{x}$, it receives a quantum $Q_{x}$ while the other classes shares $Q-Q_{x}$. First, we show that the worst-case end-to-end latency of a given class $C_{x}$ depends on the quantum $Q_{x}$ allocated to $C_{x}$ as well as on $Q-Q_{x}$, but it does not depend on the distribution of $Q-Q_{x}$ among the other classes.

Lemma 3.1. Given $Q_{x}$ and $Q$, the worst-case delay for $C_{x}$ computed by network calculus approach in [5] is the same for any quantum distribution among competing classes.

Proof. The quanta only impact the service curve for class $C_{x}$ in each switch output port. Thus we show that this service curve depends on $Q_{x}$ and $Q$, but not on quantum distribution. The service curve for class $C_{x}$ in a node is defined by equation 2 . It depends on the fraction $\rho_{x}$ of bandwidth allocated to $C_{x}$ and the DRR scheduler latency $\Theta_{x}$.

$\rho_{x}$ is defined by equation 1 , which can be rewritten

$$
\rho_{x}=\frac{Q_{x}}{Q} \times R
$$

Thus $\rho_{x}$ only depends on $Q_{x}$ and $Q$.

$\Theta_{x}$ is defined in [5] as the sum of two delays $X_{x}$ and $Y_{x}$, where,

$$
X_{x}=\frac{\sum_{1 \leq j \leq n, j \neq x}\left(Q_{j}+\Delta_{j}^{\text {max }}\right)}{R}
$$

where, $\Delta_{i}^{\max }$ is the maximum deficit for class $C_{i}$, i.e. $\operatorname{Max}_{i}-1$.

Thus, we have

$$
\begin{aligned}
& \Theta_{x}=X_{x}+Y_{x} \\
& \sum_{1 \leq j \leq n} Q_{j} \quad \sum_{1 \leq j \leq n} \Delta_{j}^{\max }
\end{aligned}
$$

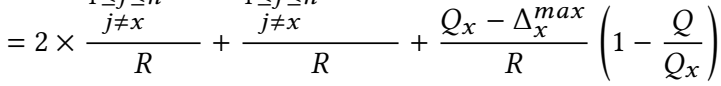

$$
\begin{aligned}
& \sum_{1 \leq j \leq n} Q_{j} \quad \sum_{1 \leq j \leq n} \Delta_{j}^{\max } \\
& =2 \times \frac{\substack{1 \leq j \leq n \\
j \neq x}}{R}+\frac{\substack{1 \leq j \leq n \\
j \neq x}}{R}+\frac{Q_{x}-\Delta_{x}^{\max }}{R}\left(\frac{Q_{x}-Q}{Q_{x}}\right) \\
& =2 \times \frac{\sum_{\substack{1 \leq j \leq n \\
j \neq x}} Q_{j}}{R}+\frac{\sum_{\substack{1 \leq j \leq n \\
j \neq x}} \Delta_{j}^{\max }}{R}+\frac{Q_{x}-\Delta_{x}^{\max }}{R}\left(-\frac{\sum_{\substack{1 \leq j \leq n \\
j \neq x}} Q_{j}}{Q_{x}}\right) \\
& \text { since } \left.\sum_{\substack{1 \leq j \leq n \\
j \neq x}} Q_{j}=Q-Q_{x}\right] \\
& =\frac{\sum_{\substack{1 \leq j \leq n \\
j \neq x}} Q_{j}}{R}+\frac{\sum_{\substack{1 \leq j \leq n \\
j \neq x}} \Delta_{j}^{\max }}{R}+\frac{\Delta_{x}^{\max }}{R}\left(\frac{\sum_{\substack{1 \leq j \leq n \\
j \neq x}} Q_{j}}{Q_{x}}\right)
\end{aligned}
$$

Therefore $\Theta_{x}$ depends on

- the link rate $R$ and the maximum deficits $\Delta_{i}^{\max }$ for each class, which does not depend on quanta,

- the quantum $Q_{x}$ assigned to class $C_{x}$ under study, 
- the sum of the quanta assigned to competing classes $\sum_{1 \leq j \leq n, j \neq x} Q_{j}$, but not the individual $Q_{j}$ values.

Based on lemma 3.1 the quantum assignment can be done in the following manner.

- the sum $Q$ of quanta allocated to classes is fixed,

- we calculate the minimum portion of $Q$ that has to be allocated to $C_{1}$ so that all the VLs in $C_{1}$ respect their deadlines (we do not need to know the quanta that will be assigned to other classes),

- we do the same for $C_{2} \ldots C_{n}$.

$Q$ value has to be large enough to lead to the valid values for each quantum (equation 3). This can be illustrated with the example in Figure 1. Let us consider three cases:

case 1: If $Q=4000$, on computing the delay bound with $\mathrm{NC}$ approach we observe that the minimum quantum required by $C_{1}$ and $C_{2}$ to respect their delay constraints are 1282 and 1119 bytes and the residual quantum for $C_{3}$ is 1599 bytes (which is $34.52 \%$ of $\mathrm{Q})$. However, this is not a valid case since the quantum assigned to $C_{1}$ should be at least 1500 bytes (equation 3 ).

case 2: Now, let us assume a higher value of $Q=6000$. In this case, the minimum quantum required by $C_{1}$ and $C_{2}$ are 2356 and 1995 bytes. The residual quantum for $C_{3}$ is 1649 bytes (27.48\%). The percentage share of quantum is clearly reduced as compared to the previous case. In the next case, let us try a value of $Q$ somewhere between that in case 1 and case 2 .

case 3: Let $Q=4500$ bytes. In this case, the minimum quantum required by $C_{1}$ and $C_{2}$ are 1507 and 1322 bytes, which gives the residual quantum for $C_{3}$ as 1671 bytes, thus, the percentage share of quantum for $C_{3}$ is increased to $37.13 \%$.

This small example shows that the ratio between computed quanta depends on $Q$. Since one goal is to minimise the response time for non critical flows (class $C_{n}$ ), we want to get the valid $Q$ value that maximises the percentage of $Q$ which is not assigned to critical classes. Next lemma states that the best $Q$ value is the smallest valid one.

Lemma 3.2. When each critical class is assigned the minimum quantum which guarantees that no deadline is missed, based on the WCTT analysis in [5], a smaller valid value of $Q$ never leads to a smaller percentage of $Q$ assigned to the non critical class $C_{n}$.

Proof. From equation 5, we have

$$
Q_{n}=Q-\sum_{1 \leq j<n} Q_{j}
$$

Therefore the percentage of $Q$ assigned to $C_{n}$ is

$$
1-\sum_{1 \leq j<n} \frac{Q_{j}}{Q}
$$

Let us consider two valid $Q$ values $Q^{\prime}$ and $Q^{\prime \prime}$ with $Q^{\prime \prime}=\sigma \times Q^{\prime}$ with $\sigma>1$. We have to show that the percentage of $Q^{\prime}$ assigned to $C_{n}$ cannot be smaller than the percentage of $Q^{\prime \prime}$ assigned to $C_{n}$. Therefore we have to show that

$$
\sum_{1 \leq j<n} \frac{Q_{j}^{\prime}}{Q^{\prime}} \leq \sum_{1 \leq j<n} \frac{Q_{j}^{\prime \prime}}{Q^{\prime \prime}}
$$

To show that this inequality is true, we show that

$$
\frac{Q_{j}^{\prime}}{Q^{\prime}} \leq \frac{Q_{j}^{\prime \prime}}{\sigma \times Q^{\prime}} \text { for } 1 \leq j<n
$$

Let us consider one class $C_{x}(1 \leq x<n)$. When the sum of quanta is $Q^{\prime}$, it gets a quantum of $Q_{x}^{\prime}$, which is the minimum quantum it needs to meet all its deadlines. When the sum of quanta is $Q^{\prime \prime}=\sigma \times Q^{\prime}$, we have to show that

$$
Q_{x}^{\prime \prime} \geq \sigma \times Q_{x}^{\prime}
$$

The worst-case delay of a $C_{x}$ frame is the maximum horizontal distance between $C_{x}$ traffic curve and $C_{x}$ service curve. $C_{x}$ traffic curve does not depend on its assigned quantum. The distance clearly decreases when $Q_{x}^{\prime \prime}$ increases. Thus inequality 13 is true if moving from a quantum of $Q_{x}^{\prime}$ out of $Q^{\prime}$ to a quantum of $\sigma \times Q_{x}^{\prime}$ out of $\sigma \times Q^{\prime}$ does not lead to a higher service curve for $C_{x}$.

The service curve for class $C_{x}$ in a node is defined by equation 2. It depends on the fraction $\rho_{x}$ of bandwidth allocated to $C_{x}$ and the DRR scheduler latency $\Theta_{x}$.

$\rho_{x}$ is defined by equation 1 . Considering $Q^{\prime}$ and $Q^{\prime \prime}$ we have

$$
\rho_{x}^{\prime}=\frac{Q_{x}^{\prime}}{Q^{\prime}} \times R \text { and } \rho_{x}^{\prime \prime}=\frac{\sigma \times Q_{x}^{\prime}}{\sigma \times Q^{\prime}} \times R
$$

Thus $\rho_{x}^{\prime}=\rho_{x}^{\prime \prime}$

Concerning DRR scheduling latency $\Theta_{x}$, we have shown in lemma 3.1 proof that

$$
\Theta_{x}^{\prime}=\frac{\sum_{1 \leq j \leq n, j \neq x} Q_{j}^{\prime}}{R}+\frac{\sum_{1 \leq j \leq n, j \neq x} \Delta_{j}^{\max }}{R}+\frac{\Delta_{x}^{\max }}{R}\left(\frac{\sum_{1 \leq j \leq n, j \neq x} Q_{j}^{\prime}}{Q_{x}^{\prime}}\right)
$$

Since $Q^{\prime \prime}=\sigma \times Q^{\prime}$ and $Q_{x}^{\prime \prime}=\sigma \times Q_{x}^{\prime}$, we have

$$
\begin{aligned}
& \Theta_{x}^{\prime \prime}= \frac{\sum_{1 \leq j \leq n, j \neq x} \sigma \times Q_{j}^{\prime}}{R}+\frac{\sum_{1 \leq j \leq n, j \neq x} \Delta_{j}^{\max }}{R}+ \\
& \frac{\Delta_{x}^{\max }}{R}\left(\frac{\sum_{1 \leq j \leq n, j \neq x} \sigma \times Q_{j}^{\prime}}{\sigma \times Q_{x}^{\prime}}\right) \\
&= \sigma \times \frac{\sum_{1 \leq j \leq n, j \neq x} Q_{j}^{\prime}}{R}+ \\
& \frac{1 \leq j \leq n, j \neq x}{R} \Delta_{j}^{\text {max }} \\
& R
\end{aligned}
$$

Since $\sigma>1$, we have $\Theta_{x}^{\prime \prime}>\Theta_{x}^{\prime}$. Thus the service curve for $C_{x}$ when considering $Q^{\prime \prime}$ and $Q_{x}^{\prime \prime}$ is under the service curve for $C_{x}$ when considering $Q_{x}^{\prime}$ and $Q^{\prime}$. This phenomenon is illustrated in Figure 4. When $\sigma$ increases, the service curve is shifted to the right, leading to a higher horizontal distance with traffic curve and, consequently, a higher worst-case delay.

Based on lemmas 3.1 and 3.2, the quantum assignment is implemented by algorithm 2 . The basic idea of the algorithm is to start from an initial value ValInit for the sum of quanta $Q$ (line 1). It computes for each critical class $C_{i}$ the minimum portion $Q_{i}$ of $Q$ that insures that no $C_{i}$ frame misses its deadline (line 9, function QuantumMin). The process stops as soon as the unused portion $Q R e s i d$ of $Q$ is smaller than the computed $Q_{i}$ value (line 10-11) or 


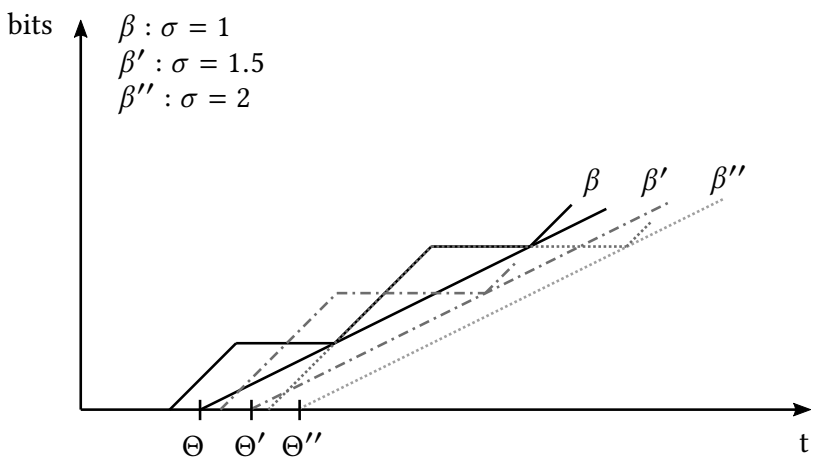

Figure 4: Service curves

all the critical classes have been treated. In the later case, the non critical class gets a quantum of QResid and a solution has been obtained. This solution might be either not valid if condition 3 is not respected (at least one class has a quantum which is smaller than its maximum frame size) or improvable if the quantum of every class exceeds its maximum frame size by more than a configured (small) value $\epsilon$ : we tolerate an $\epsilon$ difference, since it might be tricky to reach a valid solution where at least one class has its maximum frame size as quantum. In both cases (not valid or improvable solution), we adapt the sum of quanta $Q$ (lines 25-26): we increase it if the solution is not valid, we reduce it if the solution is improvable. As soon as a non improvable valid solution is found, the algorithm returns it and stops.

QuantumMin function (line 9) implements a binary search. It applies the WCTT analysis in [5] on values not greater than $Q$, until it gets the smallest value with no deadline miss.

In the next section, we illustrate Algorithm 2 on the example in Figure 1 and give some more details on the computation.

\section{QUANTUM ASSIGNMENT EXAMPLE}

The flows are divided into $n=3$ classes based on their delay constrains. $C_{1}$ and $C_{2}$ are critical flow classes with delay constraint 3100 $\mu \mathrm{sec}$ and $3200 \mu \mathrm{sec}$, respectively, and $C_{3}$ is non-critical flow class with no constraint on delay (see Table 1 ). The maximum service rate at each output port is $R=100 \mathrm{Mb} / \mathrm{s}$.

Initially, we assume a sum of quanta $Q=$ ValInit $=4550$ bytes (line 1). First critical class is $C_{1}$ (line 4). For the given sum of quanta, the minimum quantum value $Q_{i}(\mathrm{i}=1$ and 2$)$ for each class is computed (line 8-18). The function QuantumMin $(i, Q)$ computes the minimum quantum required to respect the deadline in class $C_{i}$ given the quantum sum $Q$. This computation is based on the maximum end-to-end delay in the class, obtained thanks to the WCTT analysis in [5].

The whole process is shown in Table 2 for each class of the network in Figure 1. For each class, the process is based on a binary search. In the first step, the maximum quantum is assigned to $Q_{1}$ (4550 in our case). With this quantum, the worst-case end-to-end delay for $C_{1}$ obtained by NC approach in [5] is $1007.87 \mu \mathrm{sec}$. Since, this computed delay is less than the delay constraint on $C_{1}(3100$

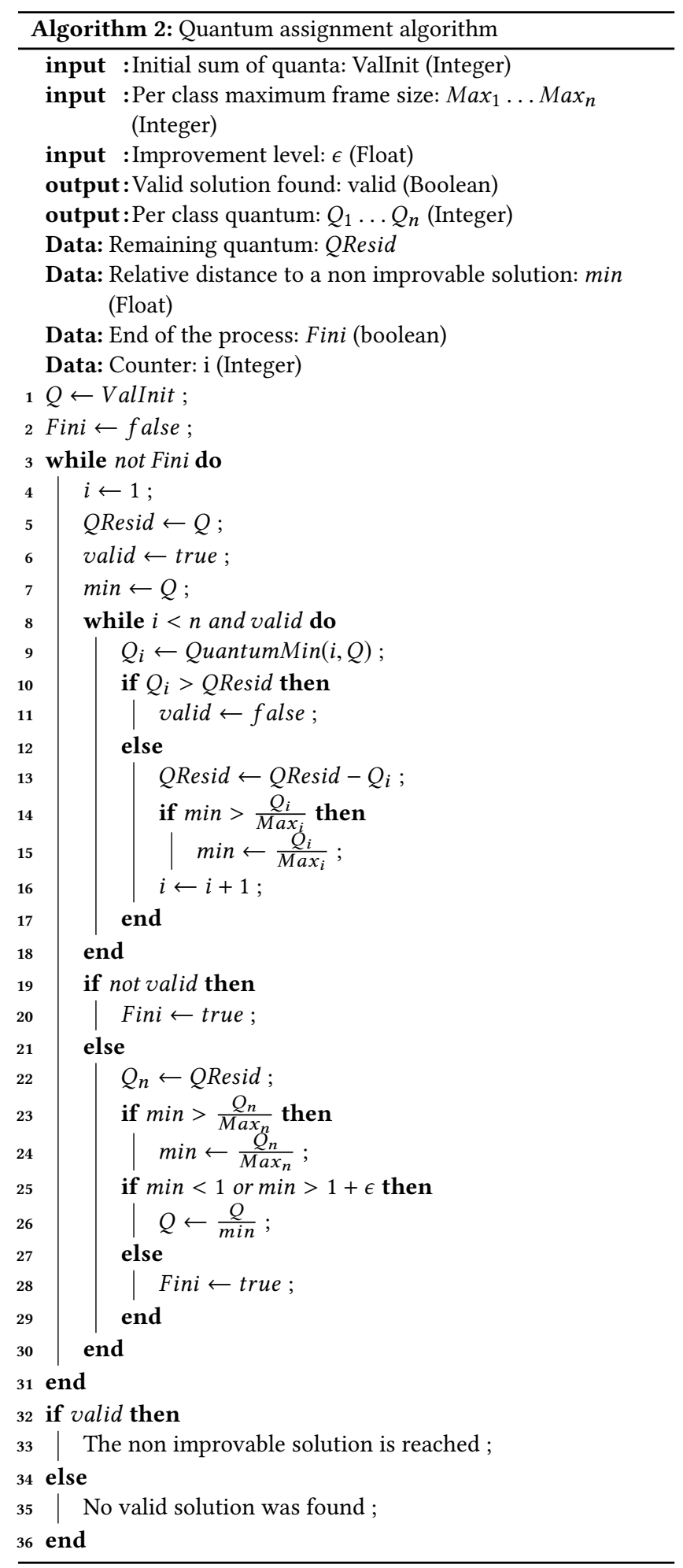


$\mu s e c), Q_{1}$ can be reduced. It will increase the worst-case delay for $C_{1}$ flows.

The delay computation in NC is based on the convergence of overall arrival curve and the service curve. Thus, the service rate $\rho_{x}$ must be more than the arrival rate $r_{x}=\sum_{v i \in C_{x}} \frac{l_{i}^{\text {max }}}{B A G_{i}}$ of the cumulative flows in $C_{x}$. In $C_{1}$, the maximum traffic arrival rate is observed at output port of switch $S_{4}$ as $6.48 \mathrm{bits} / \mu \mathrm{sec}$. If the minimum bandwidth provided to $C_{1}$ is $7.48 \mathrm{bits} / \mu \mathrm{sec}$, the corresponding quantum value is 340 bytes.

The next value assumed in binary search is the mid value between 4550 and 340, thus, $Q_{1}=2445$. The corresponding maximum end-to-end delay is $2138.72 \mu \mathrm{sec}$. Then $Q_{1}$ can be further decreased, since, the computed delay is less than the delay constraint on $C_{1}$. This process is repeated until the computed delay is close to the $C_{1}$ delay constraint (and smaller). The value returned by $\operatorname{QuantumMin}\left(C_{1}, Q\right)$ is $Q_{1}=1524$ bytes.

The residual quantum is $Q$ Resid $=4550-1524=3026$ bytes (line 13 in Algorithm 2) which can be distributed among the other classes. min is the smallest factor which is obtained from the ratio of the quantum assigned to a class and its maximum frame length. This factor will be used to optimise the quantum sum (line 26). From class $C_{1}, \min =1.016$ (line 14-16).

Next, critical class is $C_{2}$. The minimum quantum obtained from QuantumMin $\left(C_{2}, Q\right)$ is $Q_{2}=1337$ bytes (see Table 2). Thus, the residual quantum is QResid $=3026-1337=1689$ bytes. Since, the minimum quantum is successfully computed for critical classes, next class is non-critical class $C_{3}$ (line 21-30). The residual quantum can be assigned to $C_{3}$ (line 22).

Based on Lemma 3.2, the goal is to find the smallest valid value of $Q$, which we call a non improvable value.

The factor min represents the convergence towards a non improvable value of sum $Q$ of quanta. If $\min <1$, then $Q$ is lower than the expected value and if $\min >1$, then $Q$ is higher than the expected value. Thus, the non improvable sum of quanta can be obtained by the $\frac{Q}{\min }=4479$ (line 26). The distribution of this new sum of quanta among each class can be done by repeating the whole process with $Q=4479$. The corresponding values of quantum for each class is given in Table 2.

\section{EVALUATION}

In this section, we evaluate the proposed quantum assignment algorithm on an industrial-size network.

First, we perform a WCTT analysis using Network Calculus on the original industrial configuration assuming that each switch output port is controlled by a FIFO scheduler.

Then, we introduce some additional non-critical flows to this configuration and analyse the impact on the worst-case end-to-end delays of the critical flows, still considering FIFO scheduling.

Next, we assume a DRR scheduler at each switch output port. We show how the constraints on end-to-end delays for critical flows can be guaranteed while minimising the delay for non-critical flows with DRR scheduling in presence of optimised quantum values.
Table 2: Quantum assignment in the network (Figure 1).

\begin{tabular}{|c|c|c|c|c|c|c|}
\hline \multicolumn{4}{|c|}{ (bytes) } & \multicolumn{3}{|c|}{$(\mu s e c)$} \\
\hline$Q$ & $Q_{1}$ & $Q_{2}$ & $Q_{3}$ & $\max D_{C_{1}}^{E 2 E}$ & $\max D_{C_{2}}^{E 2 E}$ & $\max D_{C_{3}}^{E 2 E}$ \\
\hline \multirow{13}{*}{4550} & 4550 & - & - & 1007.87 & - & - \\
\hline & 2445 & - & - & 2138.72 & - & - \\
\hline & 1393 & - & - & 3290.62 & - & - \\
\hline & 1919 & - & - & 2614.62 & - & - \\
\hline & 1656 & - & - & 2911.66 & - & - \\
\hline & \multirow{8}{*}{1524} & - & - & \multirow{8}{*}{3088.99} & - & - \\
\hline & & 3026 & - & & 1460.72 & - \\
\hline & & 1604 & - & & 2746.07 & - \\
\hline & & 893 & - & & 4236.5 & - \\
\hline & & 1248 & - & & 3308.81 & - \\
\hline & & 1426 & - & & 2998.9 & - \\
\hline & & \multirow{2}{*}{1337} & - & & \multirow{2}{*}{3145.28} & - \\
\hline & & & 1689 & & & 3075.09 \\
\hline \multirow{13}{*}{4479} & 4479 & - & - & 1007.87 & - & - \\
\hline & 2407 & - & - & 2133.06 & - & - \\
\hline & 1371 & - & - & 3274.21 & - & - \\
\hline & 1889 & - & - & 2600.87 & - & - \\
\hline & 1630 & - & - & 2896.67 & - & - \\
\hline & \multirow{8}{*}{1500} & - & - & \multirow{8}{*}{3073.2} & - & - \\
\hline & & 2979 & - & & 1456.72 & - \\
\hline & & 1579 & - & & 2730.81 & - \\
\hline & & 879 & - & & 4217.1 & - \\
\hline & & 1229 & - & & 3290.67 & - \\
\hline & & 1404 & - & & 2982.32 & - \\
\hline & & 1316 & - & & \multirow{2}{*}{3128.82} & - \\
\hline & & 1010 & 1663 & & & 3060.18 \\
\hline
\end{tabular}

\subsection{Industrial Case Study}

We consider an industrial-size configuration. It includes 96 endsystems, 8 switches, 984 flows, and 6276 paths (multi-cast VL) [11]. We arbitrarily distribute flows between 2 critical classes: a class $C_{1}$ for flows with small BAGs (up to $16 \mathrm{~ms}$ ) and a class $C_{2}$ for flows with larger BAGs. Table 3 summarises the features of these classes.

Table 3: VL parameters in industrial configuration

\begin{tabular}{|c|c|c|c|}
\hline $\begin{array}{c}\text { Flow } \\
\text { count }\end{array}$ & $\begin{array}{c}\text { BAG } \\
\text { Range } \\
\text { (msec })\end{array}$ & $\begin{array}{c}\text { Frame } \\
\text { length } \\
\text { range } \\
\text { (bytes })\end{array}$ & Class \\
\hline $\begin{array}{c}280 \\
(1681 \text { paths })\end{array}$ & $2-16$ & $84-1497$ & $\begin{array}{c}\text { Critical } \\
C_{1}\end{array}$ \\
\hline $\begin{array}{c}704 \\
(4595 \text { paths })\end{array}$ & $32-128$ & $84-1535$ & $\begin{array}{c}\text { Less-Critical } \\
C_{2}\end{array}$ \\
\hline Additional flows & \multicolumn{3}{|c|}{} \\
\hline $\begin{array}{c}\text { Ad } \\
(120 \text { paths })\end{array}$ & 4 & $84-963$ & $\begin{array}{c}\text { Best-Effort } \\
C_{3}\end{array}$ \\
\hline
\end{tabular}

In the first step, this network is assumed to use FIFO scheduling in switch output ports (classes are not considered). Worst-case 
delays are computed for all the flows, using the classical network calculus approach for FIFO [2]. Obtained results are shown in Figure 5. In Figure 5, each unit on $\mathrm{x}$-axis represents a flow path and y-axis represents the worst-case end-to-end delay corresponding to this path. The paths are sorted in increasing order of delays in each class.
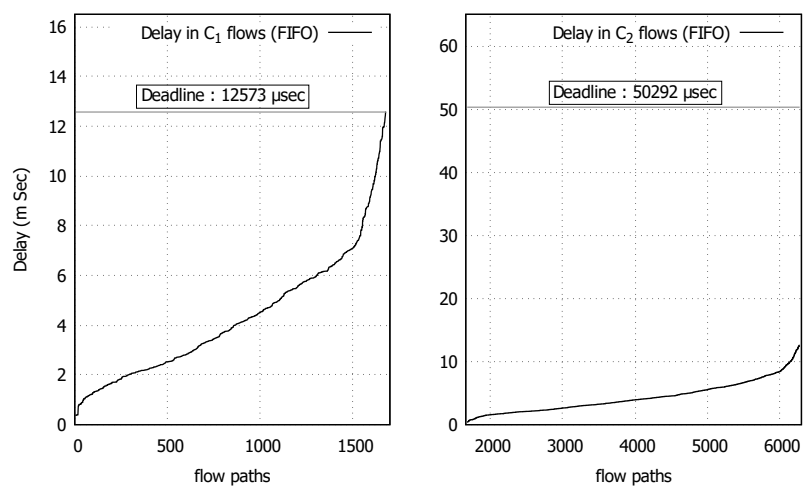

Figure 5: End-to-end delay bounds in $C_{1}$ and $C_{2}$ flows under FIFO scheduling

The maximum delays computed in the network is $12572.6 \mu \mathrm{sec}$. Therefore flow deadlines are met. Indeed deadlines for $C_{1}$ flows are $12573 \mu \mathrm{s}$, while deadlines for class $C_{2}$ flows are $50292 \mu \mathrm{s}$.

Next, we arbitrarily introduce some additional flows into this configuration. Actually, we consider a set of $40 C_{1}$ like flows. The idea is to interfere with the critical flows and evaluate the impact on worst-case end-to-end delay in the network. The added flows are considered as non-critical (best-effort) flows characterised by class $C_{3}$ shown in Table 3 .

The impact of additional flows on the critical ones is shown in Figure 6. Not surprisingly, the delay is increased for critical flows as they share waiting queues with non-critical flows. Moreover, there are 9 flows of $C_{1}$ which exceed their deadlines. The maximum delay in $C_{2}$ is still much lower than the delay constraint.
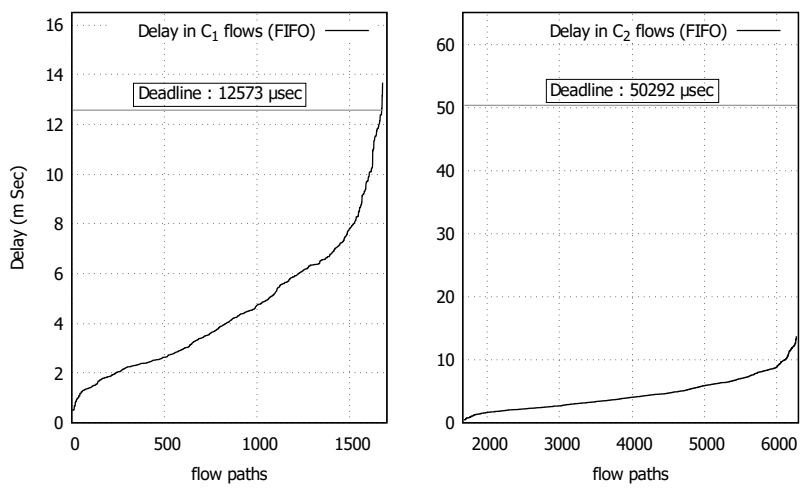

Figure 6: End-to-end delay bounds in $C_{1}$ and $C_{2}$ flows under FIFO scheduling in presence of additional flows
Now, we consider DRR scheduling at each switch output port in the given network. The DRR scheduler configuration is given in Table 4. The quantum for each class is optimised using the Algorithm 2 such that no critical flow (in $C_{1}$ and $C_{2}$ ) misses its deadline while maximising the percentage of bandwidth assigned to non-critical flows $\left(C_{3}\right)$.

Table 4: DRR configuration

\begin{tabular}{|c|c|c|}
\hline Class & $\begin{array}{c}\text { Delay } \\
\text { constraints } \\
(\mu \text { sec })\end{array}$ & $\begin{array}{c}\text { Optimized } \\
\text { Quantum } \\
\text { (bytes) }\end{array}$ \\
\hline $\begin{array}{c}\text { Critical } \\
C_{1}\end{array}$ & 12573 & $\begin{array}{c}3581 \\
\text { (Bandwidth }=56.02 \%)\end{array}$ \\
\hline $\begin{array}{c}\text { Less-Critical } \\
C_{2}\end{array}$ & $4 \times 12573$ & $\begin{array}{c}1535 \\
\text { (Bandwidth }=24.01 \%)\end{array}$ \\
\hline $\begin{array}{c}\text { Best-effort } \\
C_{3}\end{array}$ & - & $\begin{array}{c}1276 \\
\text { (Bandwidth }=19.97 \%)\end{array}$ \\
\hline \multicolumn{2}{|c|}{} \\
\hline
\end{tabular}

Based on WCTT analysis in [5], worst-case end-to-end delays are shown in Figure 7. Quantum assignment insures that critical flows are within their expected constraints and reducing one quantum for a critical class would lead to exceeded deadlines.
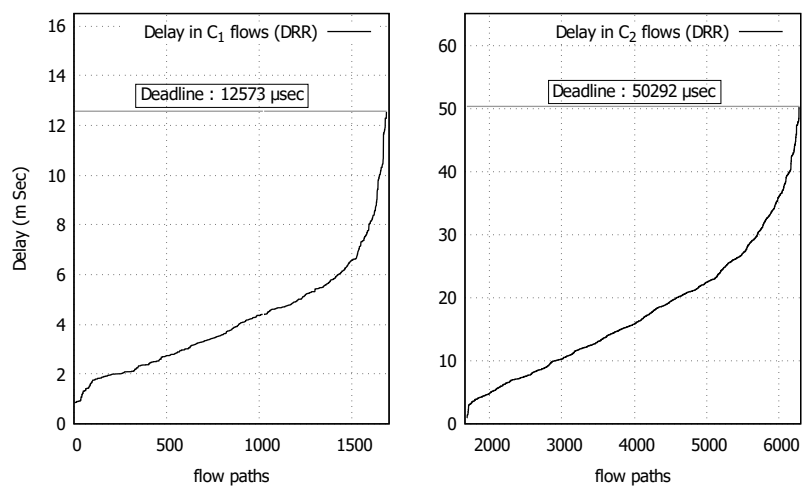

Figure 7: End-to-end delay bounds in $C_{1}$ and $C_{2}$ flows under DRR scheduling in presence of additional flows

Finally we compare the delays observed on the additional flows $\left(C_{3}\right)$ in both cases (FIFO and DRR). Worst-case delays for each flow are shown in Figure 8 and summarised in Table 5. On this specific example, DRR globally leads to smaller worst-case delays for $C_{3}$ flows than FIFO. This is due to the fact that DRR reserves bandwidth to these flows while, with FIFO, they are transmitted only when there are no pending critical frames. It would be interesting to evaluate average delays for $C_{3}$ flows, for instance by simulation. We can guess that DRR would still be better than FIFO.

\subsection{Discussion}

The NC approach for WCTT analysis used in this paper (from [5]) can be very pessimistic as it assumes that the competing classes are always active and the traffic from each class is maximum. This 

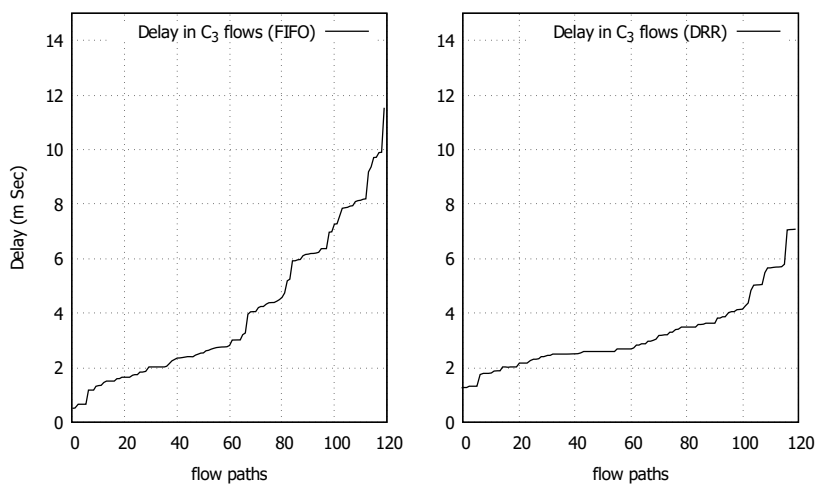

Figure 8: Comparison of end-to-end delay bounds in $C_{3}$ flows under FIFO and DRR scheduling

Table 5: Performance comparison: FIFO Vs DRR scheduling

\begin{tabular}{|c|c|c|c|c|}
\hline & \multicolumn{3}{|c|}{ Max delay ( $\mu s e c)$} & \multirow{3}{*}{$\begin{array}{l}\text { Deadlines } \\
\text { missed }\end{array}$} \\
\hline & $C_{1}$ & $C_{2}$ & $C_{3}$ & \\
\hline FIFO & 12572.6 & 12572.6 & - & \\
\hline $\begin{array}{c}\text { FIFO } \\
\text { (with added flows) }\end{array}$ & 13659.9 & 11535.4 & 11535.4 & 9 \\
\hline $\begin{array}{c}\text { DRR } \\
\text { (with added flows) }\end{array}$ & 12549.2 & 50215.7 & 7082.42 & 0 \\
\hline Deadlines & 12573 & 50292 & - & \\
\hline
\end{tabular}

pessimism also affects the optimised quantum computed by the algorithm presented in this paper, since it over-estimate worst-case delay.

The problem of pessimism in [5] was addressed in [11] by quantifying the maximum amount of traffic of each competing class within the delay bound computed by [5]. However, the optimised $\mathrm{NC}$ approach in [11] cannot be used with the quantum assignment algorithm presented in this paper as it does not respect Lemma 3.1. Indeed the optimisation in [11] depends on the quantum allocated to each competing class. Therefore the resulting WCTT analysis depends, not only on the sum of the quanta of competing classes, but also on each quantum value. Thus the algorithm proposed in this paper cannot be used.

The delay bounds computed in this paper, using [5], can be pessimistic which means that the actual delays are much lower. We evaluate the difference between both bounds on the case study. We take the optimised quanta computed earlier (Table 4) and use these quanta values in the optimised NC approach given in [11]. The results are shown in Figure 9. The maximum delay in $C_{1}$ and $C_{2}$ are 10016.9 and $24669.2 \mu \mathrm{sec}$ respectively, which are clearly much less than what was computed by the pessimistic approach. It means that we can increase the percentage of bandwidth assigned to non critical flows without compromising the constraints of critical ones.
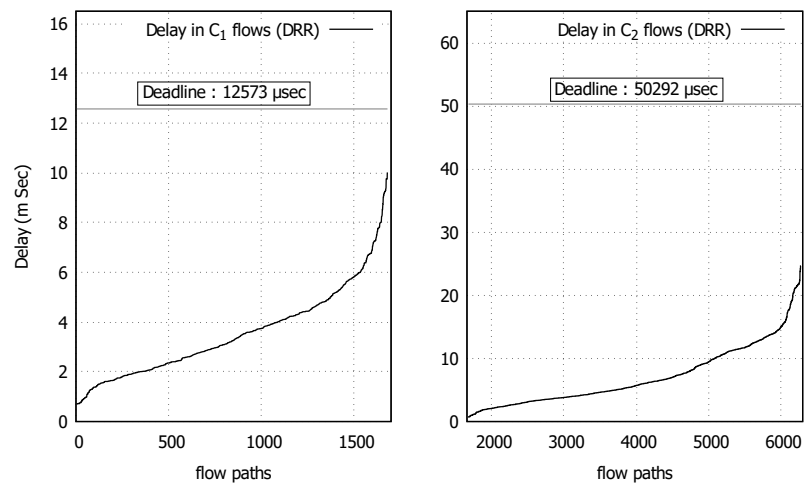

Figure 9: End-to-end delay bounds in $C_{1}$ and $C_{2}$ flows under DRR scheduling in presence of additional flows (computed by optimised NC approach)

\section{CONCLUSION}

In this paper we show how the available bandwidth of an AFDX network can be efficiently shared between critical avionics flows and non critical ones. We consider a Deficit Round Robin scheduling in switch output ports. We assume a set of critical flow classes and one non critical flow class. We propose an algorithm that assign the minimum quanta to critical classes that insure that no deadlines will be missed. Thus it maximises the percentage of bandwidth assigned to non critical flows. We show that such a strategy leads to smaller worst-case delays for non critical flows. It would be interesting to show that the trend is the same for average delays. This could be done by simulation. The other advantage of the proposed solution is to take into account the different deadlines of critical flows.

In the proposed approach, the WCTT analysis in [5] is used. It is known to be potentially very pessimistic and a much less pessimistic solution has been proposed in [11]. This optimised solution is not compatible with the algorithm proposed in this paper. Therefore one future work is to adapt the algorithm to the optimised WCTT analysis. Our intuition is that it might significantly increase the algorithm complexity.

Other scheduling policies are envisioned for QoS-aware AFDX networks, such as Weighted Round Robin [12]. Another future work is to develop a similar assignment algorithm for this scheduling policy.

\section{REFERENCES}

[1] 2002 - 2005. Aircraft Data Network, Parts 1,2,7 Aeronotical Radio Inc. Technical Report. ARINC Specification 664.

[2] Henri Bauer, Jean-Luc Scharbarg, and Christian Fraboul. 2010. Improving the Worst-Case Delay Analysis of an AFDX Network Using an Optimized Trajectory Approach. IEEE Trans. Industrial Informatics 6, 4 (Nov 2010).

[3] Jean-Yves Le Boudec and Patrick Thiran. 2012. Network Calculus: a theory of deterministic queuing systems for the internet. Vol. 2050. LNCS.

[4] Marc Boyer, Nicolas Navet, Marc Fumey, Jörn Miggie, and Lionel Havet. 2013. Combining static priority and weighted round-robin like packet scheduling in AFDX for incremental certification and mixed-criticality support. 5TH European Conference for Aeronautic and Space Sciences (EUCASS) (2013).

[5] Marc Boyer, Giovanni Stea, and William Mangoua Sofack. 2012. Deficit Round Robin with Network Calculus. Performance Evaluation Methodologies and Tools (VALUETOOLS), 2012 6th International Conference on (pp. 138-147). IEEE (October 2012), 10. 
[6] Yu Hua and Xue Liu. 2011. Scheduling design and analysis for end-to-end heterogeneous flows in an avionics network. 2011 Proceedings IEEE INFOCOM (April 2011).

[7] S. Salil Kanhere and Harish Sethu. 2002. On the latency bound of Deficit Round Robin. Computer Communications and Networks, 2002. Proceedings. Eleventh International Conference on. IEEE, 2002. p. 548-553. (October 2002), 7.

[8] Anton Kos and Saso Tomazic. 2009. A More Precise Latency Bound of Deficit Round-Robin Scheduler. Elektrotehnivski vestnik 76 (January 2009), 257-262.

[9] Sho Noda and Katsunori Yamaoka. 2016. Approach to optimal WRR weight assignment method in delay-limited environment. In 13th IEEE Annual Consumer Communications Networking Conference (CCNC). https://doi.org/10.1109/CCNC. 2016.7444945

[10] Madhavapeddi Shreedhar and George Varghese. 1996. Efficient fair queuing using deficit round-robin. IEEE/ACM Transactions on networking, 1996, vol. 4, no 3, p. 375-385 (1996), 11.

[11] Aakash Soni, Xiaoting Li, Jean-Luc Scharbarg, and Christian Fraboul. 2018. Optimizing Network Calculus for Switched Ethernet Network with Deficit Round Robin. IEEE Real-Time Systems Symposium (RTSS) (2018).

[12] Aakash Soni, Xiaoting Li, Jean-Luc Scharbarg, and Christian Fraboul. 2018. WCTT analysis of avionics Switched Ethernet Network with WRR Scheduling. 26th International Conference on Real-Time Networks and Systems (RTNS) (2018). 